\title{
Eddy Current Heating in Micro-SMES Bus-Bars
}

\author{
John M. Pfotenhauer, Jake P. Blanchard, and Carl J. Martin
}

\begin{abstract}
Results from numerical models and theoretical analyses are presented that describe the development of eddy current heating in copper bus bars, both with and without superconducting elements, in response to the rapid decay of current in a nearby superconducting magnet such as in micro-SMES systems. The analysis differs from those available in the open literature in that the change of magnetic field is neither instantaneous, nor sinusoidal. We especially investigate eddy current heating in the regime where the field decay time is on the order of the current diffusion time constant. It is found that the self-inductance of the copper bus bar contributes significantly to the time dependent heat generation. Analytical results are compared to experimental data in the form of subsequent temperature excursions of a liquid helium cooled copper bus, in response to field ramp rates on the order of 1.5 tesla/second.
\end{abstract}

Index Terms-Bus bars, eddy current heating, micro-SMES, transient film boiling.

\section{INTRODUCTION}

D URING the rapid charging and discharging of microSMES magnets, eddy currents arise in the bus bars that communicate transport current between the cryogenic and room temperature environments. If the associated dissipation is sufficiently large and long in duration, the transient heat transfer into the liquid helium bath can result in film boiling, a rapid temperature rise in the bus bar, and loss of superconductivity along the bus bars. This report explores eddy current dissipation in response to a linear field ramp (resulting from a constant voltage charge or discharge of a magnet) in conditions where the ramp time is on the same order of magnitude as the time constant associated with eddy current diffusion into the bus bar. In such conditions, a complete description of the spatiallyand time-dependent eddy currents requires a full solution of the time-dependent Maxwell equations. However, in exploring the full electro-magnetic solutions provided by the finite element software package ANSYS, a number of convenient correlations can be obtained regarding the geometric dependence of the spatially integrated eddy current dissipation.

\section{BACKGROUND}

\section{A. Analysis}

The generic problem being investigated is displayed in Fig. 1. Here a section of the bus bar connecting a superconducting so-

Manuscript received October 5, 2004. This work was supported in part by the UW-Madison Graduate School under project 010853.

J. M. Pfotenhauer is with the Applied Superconductivity Center, and the Departments of Mechanical Engineering and Engineering Physics at the University of Wisconsin-Madison, Madison, WI 53706 USA (e-mail: pfot@engr.wisc.edu).

J. P. Blanchard and C. J. Martin are with the Department of Engineering Physics at the University of Wisconsin-Madison, Madison, WI 53706 USA (e-mail: blanchard@engr.wisc.edu; cjmartin@engr.wisc.edu).

Digital Object Identifier 10.1109/TASC.2005.849340

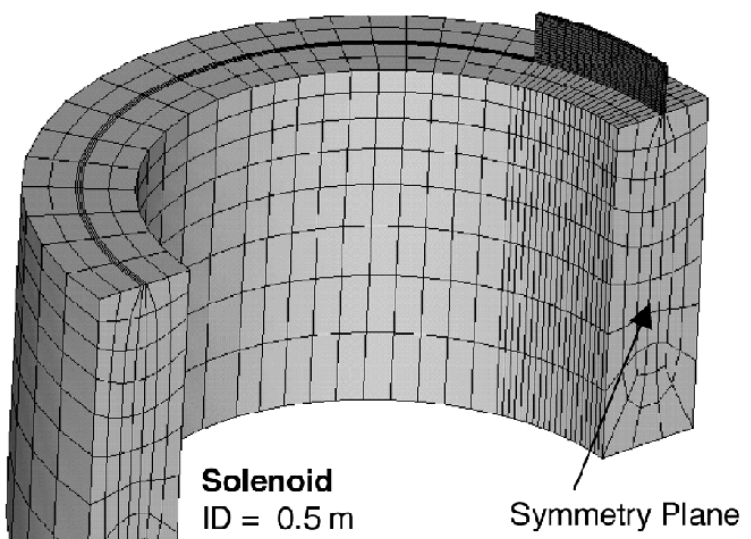
$\mathrm{OD}=0.711 \mathrm{~m}$ Height $=0.355 \mathrm{~m}$

(a)

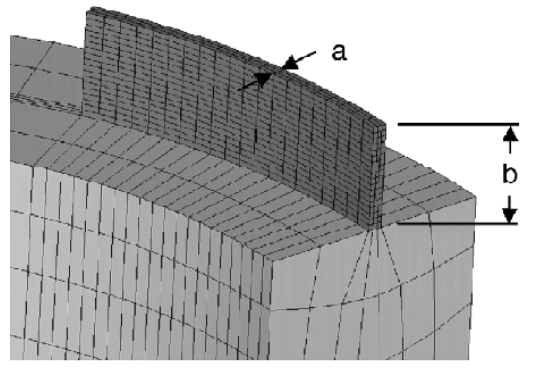

(b)

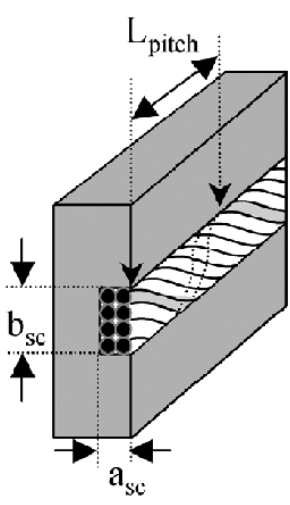

(c)
Fig. 1. Configuration of bus bar on top of solenoid magnet used in ANSYS model. (a) Solenoid details, (b) generic bus bar, (c) superconducting Rutherford cable embedded in bus bar.

lenoid to the current leads that communicate with the room temperature power system extends along the top of the solenoid in the circumferential direction. When the magnet is charged or discharged by applying a constant voltage across the current leads, a linear ramp of the current, and associated magnetic field, results. At the location of the bus bar, the field is primarily radial in direction and penetrates the bus bar in a direction that is primarily perpendicular to its face. More precisely, the field strength varies in both direction and magnitude over the complete face of the bus bar, but its primary direction is perpendicular to the face.

When the ramp rate of the magnetic field is slow compared to the time constant associated with magnetic diffusion into the bus bar, a magnetoquasistatic approximation may be used, and a simple expression is obtained for the eddy current dissipation. The time constant associated with the magnetic diffusion is given by

$$
\tau_{m}=\frac{\mu}{\rho} l^{2}
$$


where $\mu$ and $\rho$ are respectively the magnetic permeability and electric resistivity of the bus bar and $l$ is an appropriate length scale. In a homogeneous, isotropic, time invariant media, the magnetoquasistatic approximation assumes that the electric field induced by a changing magnetic field (zeroth order)

$$
\nabla \times \vec{E}=-\frac{\partial \vec{B}}{\partial t} \quad \text { (Faraday's law) }
$$

produces a 1st order magnetic field according to Ampere's law

$$
\nabla \times \vec{H}=\vec{J}+\frac{\vec{E}}{\rho_{e}}
$$

that is negligible compared to the zeroth order magnetic field. If it is also assumed that the zeroth order magnetic field is uniform and oriented only perpendicular to the face of the conducting bus $\left(\vec{B}=B_{r}\right)$, then the local power density, $\mathrm{P}^{\prime \prime \prime}(\mathrm{y})$ due to the eddy currents is found to increase quadratically in the vertical (y) direction from the conductor mid-height $(\mathrm{y}=0)$ :

$$
P^{\prime \prime \prime}(y)=\frac{\dot{B}_{r}^{2} y^{2}}{\rho_{e}}
$$

(see, for example, Iwasa [1]). An expression for the spatially averaged power dissipation per unit length associated with the eddy currents follows from this assumption in a straightforward manner

$$
P^{\prime}=\frac{a b\left(b \dot{B}_{r}\right)^{2}}{12 \rho_{e}} .
$$

Here $\mathrm{a}$ and $\mathrm{b}$ are respectively the thickness and height of the bus bar.

\section{B. Problem Specifics}

The geometry of the solenoid, and the relative location, size and orientation of the bus bar investigated in this report are as displayed in Fig. 1. The geometry and number of windings in the solenoid are chosen to produce an equivalent ramp rate (and an associated inductance) as is observed in experimental data presented later in this report. For all calculations, a $3 \mathrm{kV}$ potential is assumed across the magnet producing a linear ramp from $1300 \mathrm{amps}$ to zero in $745 \mathrm{~ms}$ and an external field ramp rate of $2.1 \mathrm{tesla} / \mathrm{s}$ at the base of the bus bar. The present study, focusing only on the eddy current behavior, does not include transport current in the bus bar. Variations in the thickness $(3.17 \mathrm{~mm}<$ $\mathrm{a}<12.7 \mathrm{~mm})$ and height $(25.4 \mathrm{~mm}<\mathrm{b}<102 \mathrm{~mm})$ of solid copper bus bars are considered, but the location relative to the solenoid remains constant for all cases. A variation on the bus bar design is also considered, in which a Rutherford-type superconducting cable is set in a groove of the copper bus bar as shown in Fig. 1(c). The bus bar length is maintained sufficiently long in all cases such that it does not influence the dissipation per unit length.

The time dependent, spatially integrated dissipation rate determined by ANSYS for the bus bar with dimensions of $\mathrm{a}=$ $6.35 \mathrm{~mm}$ and $\mathrm{b}=50.8 \mathrm{~mm}$ is shown in Fig. 2. Clearly, a steady state condition is not achieved and the magneto-quasistatic assumption may not be used here. However, the time dependence suggests an exponential rise toward a steady state condition during the ramp followed by an exponential decay after the ramp

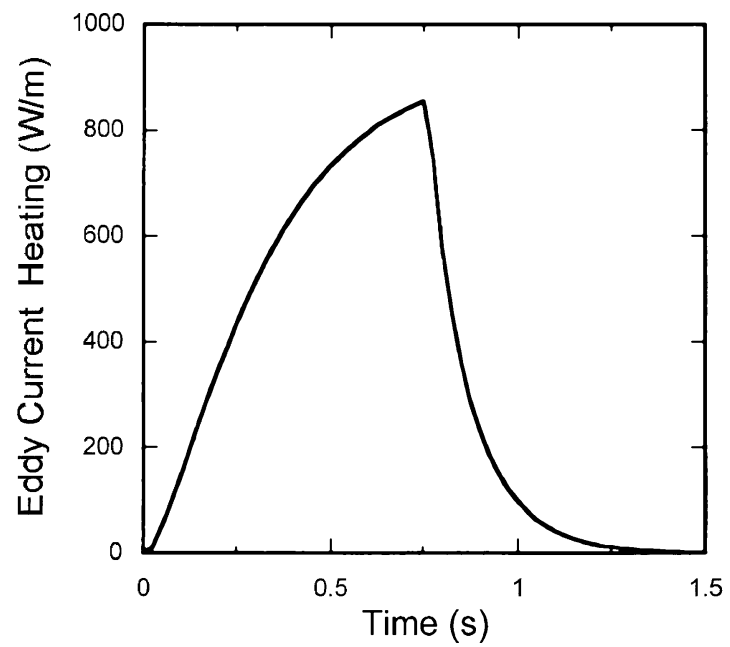

Fig. 2. Time dependent eddy current dissipation in 'baseline' bus bar geometry.

is completed. IF one assumes (as shown below, the assumption is not completely accurate), an exponential relaxation to the steady state dissipation with the relevant time constant defined by (1), then an expression for the power and total energy dissipated during the linear ramp may be obtained.

Following the approach of Iwasa [1] for the case of a bus bar of infinite extent in the $\mathrm{x}$-direction, and with thickness a (in the $\mathrm{z}$ direction) and height $b$ (in the $y$ direction) exposed to a linearly decaying magnetic field, expressions for the induced electric field and eddy current density are:

$$
\begin{aligned}
& E=y \dot{B}\left(1-e^{-\frac{t}{\tau_{m}}}\right)=y \frac{B_{0}}{t_{r}}\left(1-e^{-\frac{t}{\tau_{m}}}\right) \\
& J=\frac{y \dot{B}}{\rho_{e}}\left(1-e^{-\frac{t}{\tau_{m}}}\right)=\frac{y B_{0}}{t_{r} \rho_{e}}\left(1-e^{-\frac{t}{\tau_{m}}}\right)
\end{aligned}
$$

$\mathrm{B}_{0}$ is the initial magnetic flux density, and $\mathrm{t}_{\mathrm{r}}$ is the ramp down time from $\mathrm{B}_{0}$ to zero flux density. The time dependent eddy current power dissipation per unit length becomes

$$
\begin{aligned}
P^{\prime} & =2 a \int_{0}^{\frac{b}{2}} \frac{y^{2} \dot{B}^{2}}{\rho_{e}}\left(1-e^{-\frac{t}{\tau}}\right)^{2} d y \\
& =\frac{a b}{12} \frac{\left(b B_{0}\right)^{2}}{t_{r}^{2} \rho_{e}}\left(1-e^{-\frac{t}{\tau_{m}}}\right)^{2}
\end{aligned}
$$

Integrating (8b) over the time of the ramp provides an expression for the total eddy current heat per unit length deposited in the copper

$$
E^{\prime}=\frac{a b^{3} B_{o}^{2}}{12 t_{r}^{2} \rho_{e}}\left(t_{r}-\frac{3}{2} \tau_{m}+2 \tau_{m} e^{-\frac{t_{r}}{\tau_{m}}}-\frac{1}{2} \tau_{m} e^{-\frac{2 t_{r}}{\tau_{m}}}\right)
$$

The parenthetical terms in (9) reduce the total dissipated energy compared to the magnetoquasistatic prediction.

When a superconducting cable is integrated into the bus bar, as shown in Fig. 1(c), an additional heating term from the superconducting coupling loss must be included. An expression for the time dependent power dissipation density in the composite superconductor, in the case of the linear field ramp, is given in (12). It is derived from the dynamic equation for 
the critical-zone thickness $(\delta)$ in the composite superconductor [2], [3]:

$$
\dot{\delta}+\frac{\delta}{\tau_{o}}=\frac{2 \dot{B}_{a}}{\mu_{o} J_{s}} .
$$

For the case in which $\dot{B}_{a}=-B_{o} / t_{r}$, one finds

$$
\delta(t)=\frac{2 B_{o} \tau_{o}}{\mu_{o} t_{r} J_{s}}\left(1-e^{-\frac{t}{\tau_{o}}}\right)
$$

and the time dependent power density

$$
P^{\prime \prime \prime}(t)=\frac{\mu_{o} J_{s}^{2}}{2 \tau_{o}} \delta^{2}(t)
$$

becomes

$$
P^{\prime \prime \prime}(t)_{\text {coupling }}=\frac{\left(B_{o} L_{p}\right)^{2}}{4 \pi^{2} t_{r}^{2} \rho_{e}}\left(1-e^{-\frac{t}{\tau_{o}}}\right)^{2} .
$$

The relevant time constant associated with the coupling loss $\left(\tau_{\mathrm{o}}\right)$ is dependent on the conductor twist-pitch length, $\mathrm{L}_{\mathrm{P}}$ according to

$$
\tau_{o}=\frac{\mu}{\rho_{e}} \frac{L_{P}^{2}}{8 \pi^{2}}
$$

Multiplying (12) by the cross sectional area of the Rutherford cable, $\left(a_{\mathrm{sc}} \mathrm{b}_{\mathrm{sc}}\right)$, provides an expression for the time dependent power per unit length very similar to (8b), except that the characteristic length and time are now the twist pitch $\mathrm{L}_{\mathrm{p}}$ and $\tau_{\mathrm{o}}$ respectively, and the constant in the denominator is slightly different:

$$
P^{\prime}(t)=\frac{a_{s c} b_{s c}}{4 \pi^{2}} \frac{\left(B_{o} L_{p}\right)^{2}}{t_{r}^{2} \rho_{e}}\left(1-e^{-\frac{t}{\tau_{o}}}\right)^{2} .
$$

The total energy dissipated by this mechanism during the ramp down then has a form similar to (9).

\section{RESULTS}

\section{A. ANSYS Model}

A detailed examination of the spatial dependence of power dissipation density in the simple copper bus bar reveals that the magnetic field generated by the eddy currents contribute significantly to the time dependent field, and that the current decay in the bus bar continues after the solenoid current reaches zero (see Fig. 3, Fig. 4).

An empirical fit of the spatially dependent dissipation density reveals that it is well-described at all vertical locations by the equation

$$
P^{\prime \prime \prime} \sim\left(1-e^{-\frac{t}{0.224}}\right)^{n}
$$

For the case with $\mathrm{a}=6.35 \mathrm{~mm}$ and $\mathrm{b}=50.8 \mathrm{~mm}$ the dependence of ' $n$ ' on the vertical location is given in Fig. 4. As $n \neq 2$ everywhere, the spatially dependent power dissipation density is not captured by (8a), and the complete description does require the full time dependent solution of Maxwell's equations. However, examining the spatially integrated dissipation over the range of bus bar thicknesses, $3.17 \mathrm{~mm}<\mathrm{a}<12.7 \mathrm{~mm}$ and heights, $25.4 \mathrm{~mm}<\mathrm{b}<102 \mathrm{~mm}$ we find that it does follow

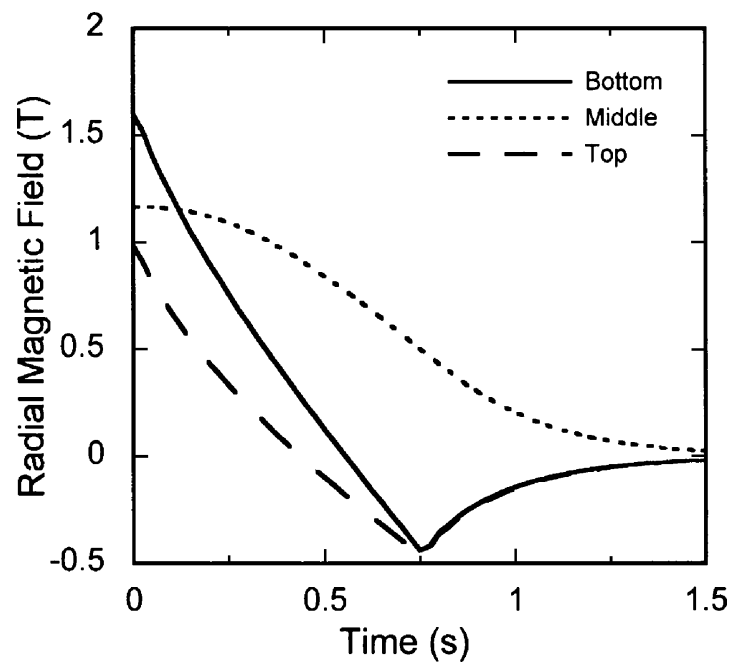

Fig. 3. Time dependent field profile in the bus bar with $\mathrm{a}=6.35 \mathrm{~mm}$ and $\mathrm{b}=50.8 \mathrm{~mm}$, resulting from a $750 \mathrm{~ms}$ ramp down of solenoid current. Bottom $(-)$, middle $(----)$, top $(---)$.

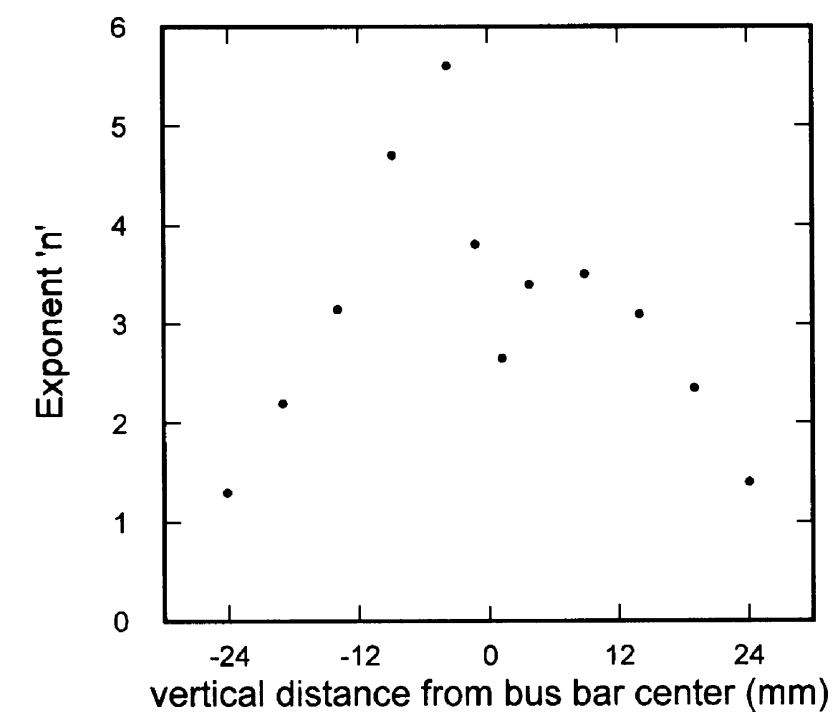

Fig. 4. Spatial variation of exponent in (15).

the form of (8b), but with a time constant $\tau_{\mathrm{ab}}$ that varies with a and $\mathrm{b}$ according to the relationship

$$
\tau_{a b}=\frac{\mu_{0}}{\rho} \frac{a^{0.81} b^{1.15}}{19.78}
$$

which is within the fit-accuracy of the usual length-squared dependence of the magnetic diffusion time constant. At present it is unclear why the denominator in (16) is a factor of 5 larger than would be defined by the half-lengths of $a$ and $b$.

\section{B. Significance for Thermal Runaway}

Using $\tau_{\mathrm{ab}}$ given by (16) for $\tau_{\mathrm{m}}$ in the expression for the power and energy dissipation per unit length, (8a) and (9), one finds a significant dependence of $\mathrm{P}$ and $\mathrm{E}$ on the aspect ratio of the bus bar. For example, using the dimensions $\mathrm{a}=6.35 \mathrm{~mm}$ and $\mathrm{b}=50.8 \mathrm{~mm}$ as a baseline dimension, Fig. 5 displays the relative change in dissipation energy for other combinations of a 


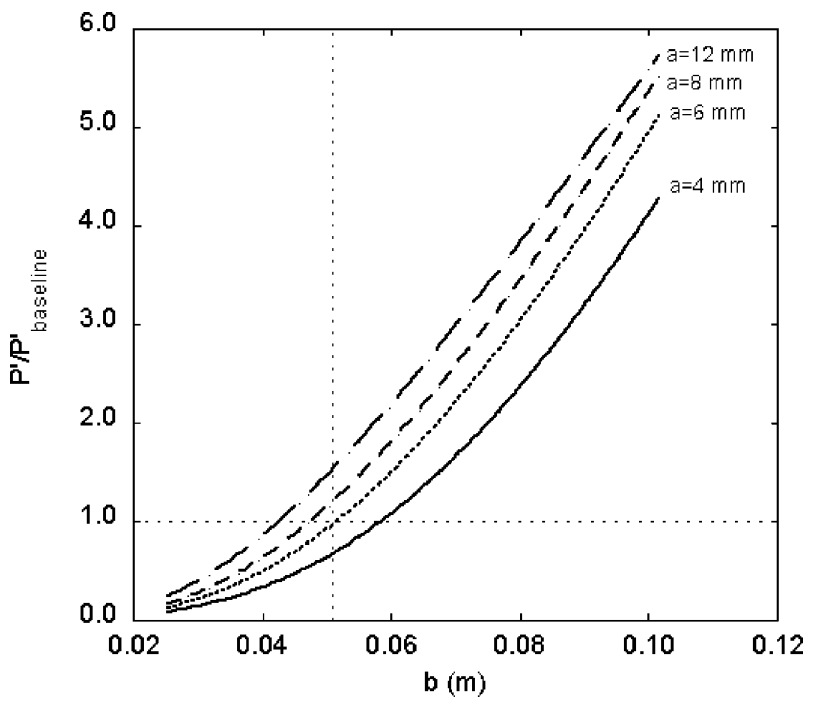

Fig. 5. The ratio of power dissipation per unit length at the end of the $745 \mathrm{~ms}$ ramp as a function of the bus bar dimension.

and $\mathrm{b}$ within the range investigated here. Also, the spatial dependence of the power dissipation described by Fig. 4 results in local power dissipation that exceeds the average by up to a factor of 5 for the dimensions considered in this report. The significance of these observations is reflected in a set of related experiments.

Thermal measurements at the center of the face of various bus bar segments were gathered under conditions such as depicted in Fig. 1 and in response to the linear field ramp studied here. Sample 1 (baseline sample) is a copper bus bar with dimensions $\mathrm{a}=6.35 \mathrm{~mm}$ and $\mathrm{b}=50.8 \mathrm{~mm}$. Sample 2 is comprised of two copper bars of dimensions $\mathrm{a}=3.175 \mathrm{~mm}$, $\mathrm{b}=76.2 \mathrm{~mm}$ that are sandwiched together with a thin insulating strip between them. Sample 3 has the same overall dimensions as sample 1 , but includes a superconducting cable with dimensions $\mathrm{a}_{\mathrm{sc}}=3.175 \mathrm{~mm}$ and $\mathrm{b}_{\mathrm{sc}}=19.1 \mathrm{~mm}$. The time dependent temperatures measured on the face of the three samples are shown in Fig. 6.

In all cases, the time constant associated with thermal diffusion in the samples is on the order of $1 \mathrm{~ms}$ and it is reasonable to assume that although the dissipation varies significantly over the height of the bus bar, the temperature is spatially averaged throughout the test. Transient heat flux data from Steward [4] for vertically oriented heaters in a saturated helium bath display a transition to film boiling after $10 \mathrm{~ms}$ when the surface heat flux is $1 \mathrm{~W} / \mathrm{cm}^{2}$ or larger and an associated temperature rise above $14 \mathrm{~K}$. For heat flux values less than $0.6 \mathrm{~W} / \mathrm{cm}^{2}$, no tran-

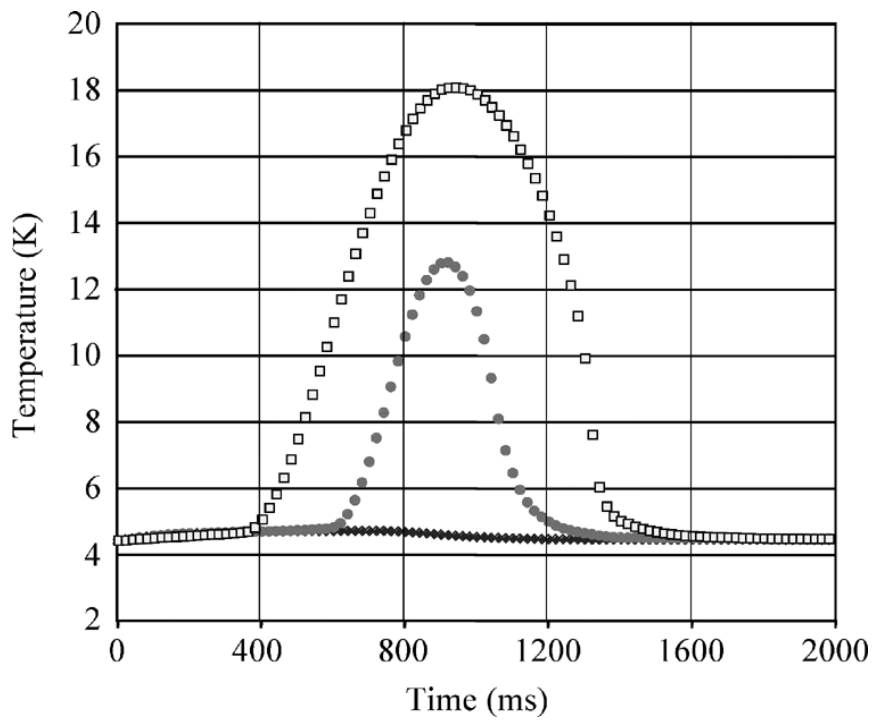

Fig. 6. Temperature response of helium bath cooled bus bars to $745 \mathrm{~ms}$ linear ramp. Sample 1: $\diamond$; sample 2: $\square$; sample 3: •.

sition to film boiling is observed and the surface temperature remains below $5 \mathrm{~K}$. The peak surface heat-flux values predicted by the ANSYS model; $0.83 \mathrm{~W} / \mathrm{cm}^{2}$ for sample $1,1.1 \mathrm{~W} / \mathrm{cm}^{2}$ for sample 2 (including the additional dissipation from the superconducting cable), and $1.4 \mathrm{~W} / \mathrm{cm}^{2}$ for sample 3 , combined with Steward's data, provide a plausible explanation for the associated temperature-response displayed in Fig. 6.

\section{SUMMARY}

A fully time-and-spatially dependent solution to Maxwell's equation is necessary to accurately describe the time dependent eddy current dissipation in a micro-SMES bus bar when the magnetic diffusion time constant is comparable to the ramp time. However, a simple exponential relaxation model captures the primary features of the time- and geometry-dependent dissipation.

\section{REFERENCES}

[1] Y. Iwasa, Case Studies in Superconducting Magnets. New York: Plenum, 1994, pp. 43-44.

[2] W. J. Carr Jr., "AC loss in a twisted filamentary superconducting wire," J. Appl. Phys., vol. 45, no. 2, pp. 929-934, Feb. 1974.

[3] - "Conductivity, permeability, and dielectric constant in a multifilament superconductor," J. Appl. Phys., vol. 46, no. 9, pp. 4043-4047, Sep. 1975.

[4] G. Steward, "Transient helium heat transfer phase 1, static coolant," Int. J. Heat Mass Transfer, vol. 21, p. 863, 1978. 